\title{
Effects of transcranial direct current stimulation on gait in people with Parkinson's disease: study protocol for a randomized, controlled clinical trial
}

\author{
Vida Alizad ${ }^{1,2^{*}}$, Marcus Meinzer ${ }^{3}$, Laurent Frossard ${ }^{4}$, Remco Polman ${ }^{5}$, Simon Smith ${ }^{6}$ and Graham Kerr ${ }^{1}$
}

\begin{abstract}
Background: Gait difficulties are common and frequently devastating to people with Parkinson's disease (PD). These difficulties are often followed by an increased risk of falls, leading to injury, hospitalization and mortality. The dysfunction in the basal ganglia-thalamocortical motor circuits and reduced activity in the premotor and primary motor cortices has raised interest in transcranial direct current stimulation (tDCS) as an adjunct intervention in PD. tDCS might provide a potentially safe and non-invasive treatment by modulating cortical excitability and behavioural outcomes. The aim of this study is to compare the effects of different monopolar and bipolar montages of tDCS administered to the motor cortex and cerebellum on gait speed in PD.
\end{abstract}

Methods: This study will be conducted in a randomized, double-blind cross-over design. Eighteen participants diagnosed with Parkinson's disease will receive anodal and sham tDCS $\left(1 \mathrm{~mA}, 20 \mathrm{~min}, 10 \times 4 \mathrm{~cm}^{2}\right)$ over the premotor and primary motor cortices with the cathode over the cerebellum during treadmill walking. Three montages will be applied over three sessions and compared: anodal tDCS with a small active cathode $\left(4 \times 4 \mathrm{~cm}^{2}\right)$; anodal tDCS with a large, functionally inert cathode $\left(10 \times 10 \mathrm{~cm}^{2}\right)$; and sham tDCS. The primary outcome measure is gait speed, and secondary outcome measures include gait parameters (temporospatial, segmental, kinematic), the Timed Up and Go test and lower limb muscle activity patterns as measured by electromyography.

Discussion: This study will investigate the short-term effects of anodal tDCS over the premotor and primary motor cortices on gait abilities using monopolar and bipolar montages in people with PD. The outcomes will inform future studies aimed at inducing longer-lasting changes in neural excitability and performance using multisession tDCS designs in PD.

Trial registration: Australian New Zealand Clinical Trials Registry (ANZCTR), ACTRN12618000063213. Registered on 17 January 2018. Retrospectively registered.

Keywords: Parkinson's disease, Transcranial direct current stimulation, Gait

\footnotetext{
*Correspondence: v.alizad@qut.edu.au; vi.alizad@uswr.ac.ir

${ }^{1}$ Movement Neuroscience Program, Institute of Health and Biomedical Innovation, Queensland University of Technology, Brisbane, QLD, Australia ${ }^{2}$ Iranian Research Centre on Ageing, The University of Social Welfare and rehabilitation Sciences, Tehran, Iran

Full list of author information is available at the end of the article
}

(c) The Author(s). 2018 Open Access This article is distributed under the terms of the Creative Commons Attribution 4.0 International License (http://creativecommons.org/licenses/by/4.0/), which permits unrestricted use, distribution, and reproduction in any medium, provided you give appropriate credit to the original author(s) and the source, provide a link to the Creative Commons license, and indicate if changes were made. The Creative Commons Public Domain Dedication waiver (http://creativecommons.org/publicdomain/zero/1.0/) applies to the data made available in this article, unless otherwise stated. 


\section{Background}

Gait difficulties are common and often incapacitating for individuals with Parkinson's disease (PD). They manifest as reduced gait speed, stride length, coordination and arm swing [1] as well as increased cadence, upper body movement and double support duration $[2,3]$. Underpinning this altered gait are changes in muscle activation. This includes increased activation of hamstrings and quadriceps in mid-stance and late stance phases and decreased activation of tibialis anterior and gastrocnemius lateralis muscles in early stance phase, which results in delayed heel-off phase and gait initiation $[4,5]$. Importantly, gait deficiencies result in a nine-times greater risk of falls [6], leading to a five-times greater risk of sustaining fall-related injuries, hospitalization and mortality for people with PD [3].

Motor complications in PD have been linked to degeneration of dopaminergic neurons in the substantia nigra compacta of the basal ganglia, which results in a deficit of dopamine and disruption of dopaminergic neurotransmission [7]. There is a consequent dysfunction in the basal ganglia-thalamocortical motor circuits with an overactivity of inhibitory efferents from the basal ganglia to the thalamus, resulting in suppression on thalamocortical projections, with abnormal activity in the premotor and primary motor cortices, resulting in gait difficulties $[8,9]$. The dopamine deficit and dysfunction in the basal ganglia-thalamocortical motor circuits also affect cortico-cerebellar circuits as they are functionally connected $[10,11]$. The cerebellum has been shown to be hyperactive in PD, presumably to compensate for the deficiency in function of the basal ganglia and the cortico-cerebellar circuit [10].

Non-invasive brain stimulation of the premotor and primary motor cortices may modulate neural activity in these neural circuits $[10,12,13]$ and re-equilibrate the cortico-cerebellar circuits [14], thereby improving gait ability and lower limb muscle activity in people with PD.

Transcranial direct current stimulation (tDCS) is a non-invasive brain stimulation technique now widely used in neuroscience and clinical research in humans $[15,16]$. It has an excellent safety profile [17] and is a low-cost technique suited for double-blind clinical trials [18]. The tDCS process modulates cortical excitability via a weak direct current that is delivered by two or more scalp-affixed electrodes [19]. Anodal tDCS (a-tDCS) typically increases cortical excitability and cathodal tDCS (c-tDCS) decreases it [13]. The underlying mechanisms by which tDCS modulates neurophysiology and behaviour are yet to be fully understood [20]. However, acute effects of a single session (e.g. 20$30 \mathrm{~min}$ ) might result in transient modulation of the neural resting membrane potential $[19,21]$. Further, the cumulative effects of repeated tDCS sessions can be explained by modulation of post-synaptic connections, similar to long-term potentiation and long-term depression, which play a key role in neuroplasticity underlying adaptive human behaviour and learning [19].

Emerging research suggests the potential for a-tDCS over the primary motor cortex (M1) to improve gait difficulties in PD due to the dense connectivity of the cortex and the basal ganglia, and the possibility of targeting the basal ganglia-thalamocortical motor circuits [22-27]. However, results from previous studies have indicated that the effects of tDCS on gait speed [24, 26-34] and stride length [24, 27, 32] have been variable and appear to be dependent on stimulation site and electrode size. In one study, bilateral a-tDCS $\left(3.5 \times 5 \mathrm{~cm}^{2}\right)$ of either premotor and motor or prefrontal cortices with the cathode $\left(5 \times 5 \mathrm{~cm}^{2}\right)$ over the mastoid demonstrated significant improvement in gait speed [28]. The most substantial gait improvement was reported by Kaski et al. (2014) after applying bilateral a-tDCS combined with physical training or tango [33, 35]. This bilateral arrangement was employed because gait control is the result of parallel and bilateral involvement of motor and premotor cortices; the authors used a bilateral stimulation set-up, and the anode $\left(10 \times 4 \mathrm{~cm}^{2}\right)$ was centred over the premotor and primary motor cortices. The cathode $\left(4 \times 4 \mathrm{~cm}^{2}\right)$ was attached over the inion to ensure current flow through the sensorimotor strip but without affecting the cerebellum. While this montage showed beneficial effects on gait speed and stride length, it remained unclear whether these effects were mediated by sensorimotor effects of the anode, possible inhibitory effects on the cerebellum due to the proximity of the cathode to this region or synergetic effects induced by the bipolar set-up. Therefore, a systematic investigation of electrode size and placement, which most likely determine the extent of tDCS effects on gait abilities in PD, is required.

In the present study, we will address this issue by systematically investigating the effects of a-tDCS over the premotor and primary motor cortices and the synergetic effects of a-tDCS over the premotor and primary motor cortices and c-tDCS over the cerebellum. As it is thought that tDCS is most effective when combined with a behavioural task [36], tDCS will be administered during simultaneous treadmill walking, because a recent study reported more pronounced effects after this task compared to other physical tasks in PD [37]. This ensures that the neural circuits, and particularly M1, are active during application of the tDCS. In this study, we also took advantage of the same anode electrode parameters used in studies by Kaski et al. [30, 33, 35, 38]. Applying bilateral a-tDCS over the premotor and primary motor cortices and comparing the effects of both active (small) and functionally inert (large) cathodes over the cerebellum in PD could disentangle the location of 
possible tDCS effects on gait abilities in PD. We hypothesize that a large cathode will render stimulation over the cerebellum inactive (i.e. a monopolar M1 set-up), whereas a smaller cathode will exert additional inhibitory effects on the cerebellum (i.e. a bipolar M1 cerebellar set-up) [39]. To our knowledge, no study to date has analysed the gait parameters and electromyography (EMG) of the lower limb focusing specifically on different bilateral tDCS montages.

Thus, this randomized placebo ("sham tDCS)" controlled study aims to examine the short-term effects of (1) monopolar bilateral a-tDCS over the premotor and primary motor cortices (exploratory aim 1); (2) bipolar bilateral a-tDCS over the premotor and primary motor cortices and cerebellum cortex (exploratory aim 2) while walking on a treadmill on gait speed as the main outcome measure and other gait kinematics and EMG in people with PD when optimally medicated. The findings will be reported and disseminated through peer-reviewed journal publication and conference presentations. The results of the study will also be presented for PD support groups who contributed in participant recruitment.

\section{Methods}

\section{Study design and outline}

The study will employ a randomized, double-blind, sham-controlled cross-over design to assess the effect of a-tDCS delivered during treadmill walking on natural overground gait performance and associated muscle activation. Participants will receive bilateral active or sham tDCS for 20 min while walking on a treadmill over three sessions. Gait will be assessed using a three-dimensional (3D) motion capture system and EMG before and after applying tDCS in each session. All assessment will be conducted by the principal researcher, who was trained for conducting all the assessments. The overall study design is illustrated in Fig. 1. A Standard Protocol Items: Recommendations for Interventional Trials (SPIRIT) schedule is presented in Fig. 2, and a SPIRIT checklist is available in Additional file 1. The steering committee is the supervisory panel for the principal researcher, and data monitoring is done by the Research Method Advisory Group in the Institute of Health and Biomedical Innovation (IHBI) at Queensland University of Technology (QUT). The trial will be monitored on a weekly basis by the supervisory panel. The Institutional Human Research Ethics Committee approved the research. The ethics committee will be informed of any trial modifications, and these updates will be made to the trial information on the Australian New Zealand Clinical Trials Registry website. The study will be undertaken and reported based on the Consolidated Standards of Reporting Trials (CONSORT) statement for non-pharmacological treatment.

\section{Participants}

Eighteen participants diagnosed with idiopathic PD aged 40-80 years old will be recruited from (1) Parkinson's Queensland Incorporated (PQI), i.e. a community-based PD advocacy and support group; (2) local neurology clinics; (3) PD support groups; (4) advertisement via university website, emails and Facebook. Potential participants will be sent an information sheet explaining the details of the study and an invitation to contact one of the research team members if they wish to participate in the study.

Interested participants will initially be screened for the basic exclusion criteria, contraindications and special considerations before tDCS via a telephone screening procedure. Exclusion criteria will compromise the presence of the following: uncorrected vision impairment, heart failure, uncontrolled blood pressure, cardiovascular disease, asthma, vertigo, frequent falls, dizziness, musculoskeletal injuries within the last 18 months and orthopedic surgery within the last 12 months. The tDCS exclusion criteria include having a previous adverse reaction to transcranial magnetic stimulation (TMS)/tDCS, seizure, unexplained loss of consciousness, stroke, serious head injury, surgery to the head, any brain-related neurological illness other than PD, any illness that may have caused brain injury, frequent or severe headache, having any metal in the head, having any implanted medical device, being pregnant and having any epileptic family members. Participants meeting basic inclusion criteria for participation in the study will be scheduled to visit the movement assessment laboratory, provided with full participant information by the principal researcher on the nature of the study and asked to provide written informed consent. They will undergo a clinical eligibility screening as well as baseline clinical assessments at the IHBI, QUT. For testing at the IHBI, emergency procedures will be followed as per QUT policy. QUT provides for limited counselling services (face-to-face only) for research participants of QUT projects who may experience discomfort or distress as a result of their participation in the research. Once recruited into the study, participants will be sent reminders via emails and text messages to ensure adherence to attending the three testing sessions. Participants will be asked not to start a new intervention until they complete the trial. However, if they wish to, they will be permitted to do so, and any new intervention will be documented.

\section{Clinical eligibility screening}

Clinical assessments will test (1) cognitive function using Addenbrooke's Cognitive Examination Revised (ACE-R), whereby a score $<82$ indicates likely dementia [40]; (2) disease severity using the Movement Disorder Society Unified Parkinson's Disease Rating Scale (MDS-UPDRS), where the total UPDRS score is determined as the sum 


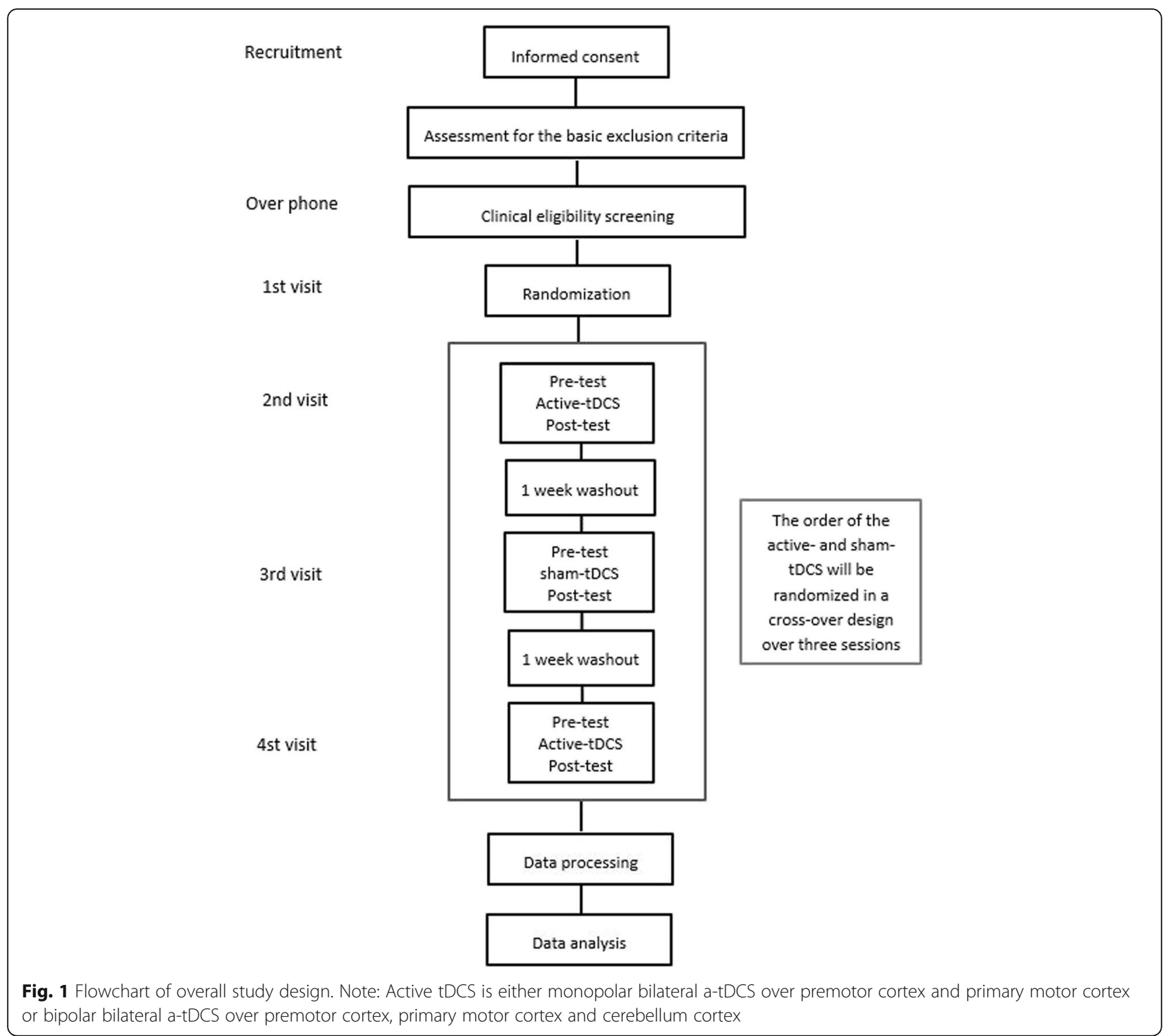

of the scores of all items. Each item can be rated with scores of $0=$ normal to $4=$ severe; the higher the total score, the more severe the disease [41].

\section{Clinical baseline assessment}

Eligible participants will be asked to perform further clinical assessments, including:

1. Visual function including contrast sensitivity and visual acuity. Contrast sensitivity will be assessed using the Pelli-Robson chart with normal values of $1.80-2.10$ at both 1 and $3 \mathrm{~m}$ distance for persons $40-59$ years old, and $1.65-1.95$ at $1 \mathrm{~m}$ and $1.65-$ 2.10 at $3 \mathrm{~m}$ for those $\geq 60$ years old [42] ; and the Melbourne Edge Test, where abnormal values differ for different age groups ranging from $<22$ in individuals younger than 50 years to $<14$ in individuals older than 80 years old [43]. Visual acuity will be assessed using the Bailey-Lovie chart set, where the values of the logarithm of the minimum angle of resolution (logMAR) range from normal $(-0.3)$ to poor $(1.00)[44,45]$.

2. Lower limb peripheral sensation (internal and external malleolus, distal phalanx of third toe, arch of foot, heel and dorsum of proximal phalanx of toe) will be assessed using a Semmes-Weinsteintype pressure aesthesiometer; the lower tactile threshold score, the better [46].

3. Mobility will be assessed using the Tinetti balance and gait test, whereby a value $\leq 18$ suggests high risk for falls and $\geq 24$ suggests low risk for falls [47].

Following the clinical assessments, questionnaires will be provided to the participants to be completed at their 


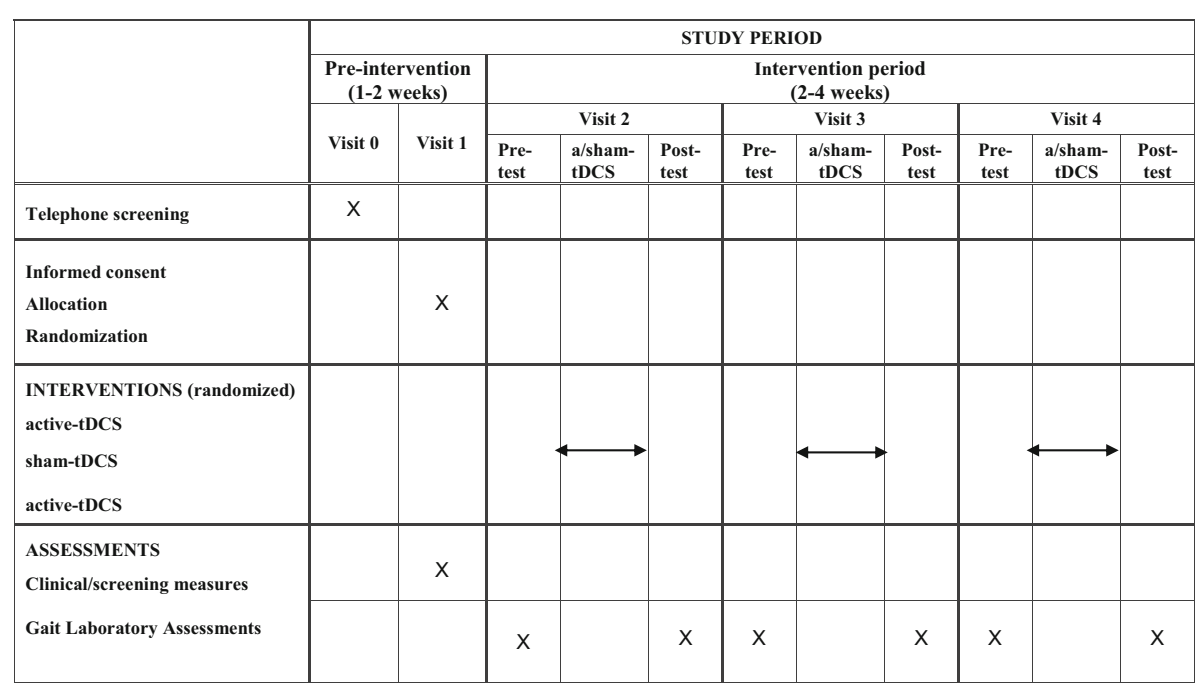

Fig. 2 SPIRIT schedule

convenience at home, including demographic information, the International Physical Activity Questionnaire (IPAQ) [48], the Activities-specific Balance Confidence (ABC) Scale [49], a medication diary to calculate levodopa equivalent daily dose, quality of life questionnaires (Parkinson's Disease Questionnaire (PDQ-39) [50], Parkinson's diseases gait and falls questionnaire [51] and the UPDRS (Part 2: activities of daily living; patient questionnaire section) [52]. All questionnaires have good psychometric properties and have been used in previous PD trials [3].The participants will be asked to attend the gait laboratory for three visits with at least a 1-week interval between them and to return the completed questionnaires on the first visit. The assessments and the time points and all outcome measures at which the assessments will be taken are presented in Table 1. All questionnares and forms will be found in the online thesis, which will be available via the QUT library.

\section{Primary and secondary outcomes}

The primary outcome will be gait speed, and secondary outcomes include other gait kinematics, muscle activation and functional mobility (Table 1).

\section{Pre- and post-tDCS assessments}

Three-dimensional gait assessment Objective gait evaluation will be performed using a typical 3D assessment relying on motion capture [53, 54]. Gait speed as the primary outcome and all kinematic data will be collected using the Vicon system (Nexus 2.6; Vicon, Oxford, UK) including 12 cameras recording markers at $200 \mathrm{~Hz}$ within a calibration volume (e.g. $\mathrm{L}: 9.3 \times \mathrm{W}: 2.6 \times \mathrm{H}: 2.5 \mathrm{~m})$.
A total of 39 spherical markers $(14 \mathrm{~mm})$ will be positioned over body landmarks, based on a modified Helen Hayes marker set [3], including the head (forehead and back of the head); trunk (C7 spinus process, tenth thoracic vertebra, right scapula, jugular joint and xiphoid process); hips (anterior superior Iliac spine and posterior superior iliac spine); upper limbs (lateral border of acromion, upper arm, olecranon process of the humerus, lower arm, radial and ulnar styloids and second metacarpal head); and lower limbs (thigh, tibia, knee, ankle, second metatarsal head). Markers will be attached directly onto the skin using double-sided and hypafix tape. Marker placement will be performed by the same researcher in each session to minimize inter-tester variability. The $3 \mathrm{D}$ position of the markers will be expressed in the Global Coordinate System (GCS) placed approximately $1.3 \mathrm{~m}$ on the right side and $4.6 \mathrm{~m}$ behind the centre of the $10-\mathrm{m}$ straight walkway.

First, the participant will be asked to walk naturally 10 times over the walkway to establish his/her self-selected walking speed. This speed will be used to set the treadmill speed during the tDCS sessions. Next, the participant's anthropometrics will be measured and entered into the Vicon Nexus software, including body mass, height, leg length, knee and ankle width, elbow and wrist width and hand thickness.

Then, an initial recording of the participant standing still in the middle of the capture volume will be conducted for static calibration to facilitate automated detection and tracking of markers. Finally, the participant will be asked to walk six times in a straight line, over even and uneven surfaces on the walkway located in the middle of the calibration volume. Sufficient rest will be allowed in between trials to avoid participant's fatigue. 
Table 1 Baseline and follow-up outcome measures

\begin{tabular}{|c|c|c|c|c|c|}
\hline \multirow[t]{2}{*}{ Outcome measure } & \multirow[t]{2}{*}{ Instrument } & \multirow{2}{*}{$\begin{array}{l}\text { To be completed } \\
\text { at convenient time }\end{array}$} & \multicolumn{2}{|l|}{ Visit 1} & \multirow{2}{*}{$\begin{array}{l}\text { Visits } \\
2-4\end{array}$} \\
\hline & & & Screening & Clinical & \\
\hline \multicolumn{6}{|l|}{ Clinical/screening measures } \\
\hline Cognitive function & Addenbrooke's Cognitive Examination (ACE-R) & & $\checkmark$ & & \\
\hline Disease severity & $\begin{array}{l}\text { Unified Parkinson's Disease Rating Scale } \\
\text { (UPDRS) and Hoehn and Yahr scale }\end{array}$ & & $\checkmark$ & & \\
\hline \multicolumn{6}{|l|}{ Visual function } \\
\hline Visual acuity & Binocular visual acuity: Bailey-Lovie & & & $\checkmark$ & \\
\hline \multirow[t]{2}{*}{ Contrast sensitivity } & Pelli-Robson contrast sensitivity chart & & & $\checkmark$ & \\
\hline & Melbourne Edge Test & & & $\checkmark$ & \\
\hline Touch sensitivity & Semmes-Weinstein monofilament test & & & $\checkmark$ & \\
\hline Clinical balance and gait & Tinetti Gait and Balance Instrument & & & & $\checkmark$ \\
\hline Mobility & Timed Up and Go (TUG) test & & & & $\checkmark$ \\
\hline \multicolumn{6}{|l|}{ Gait characteristics } \\
\hline Spatiotemporal parameters & Nexus 2.6; Vicon, Oxford, UK & & & & \\
\hline Gait speed $(\mathrm{m} / \mathrm{s})$ & & & & & $\checkmark$ \\
\hline Cadence (step/min) & & & & & $\checkmark$ \\
\hline Spatial parameters $(\mathrm{cm})$ & Nexus 2.6; Vicon, Oxford, UK & & & & \\
\hline Stride length & & & & & $\checkmark$ \\
\hline Step length & & & & & $\checkmark$ \\
\hline Step width & & & & & $\checkmark$ \\
\hline Maximum toe clearance & & & & & $\checkmark$ \\
\hline Temporal parameters (s and \% GC) & Nexus 2.6; Vicon, Oxford, UK & & & & \\
\hline $\begin{array}{l}\text { Stride/stance/swing/single support/double } \\
\text { support/GC time }\end{array}$ & & & & & $\checkmark$ \\
\hline Segmental linear motion $(\mathrm{cm})$ & Nexus 2.6; Vicon, Oxford, UK & & & & \\
\hline Centre of mass (COM) VT/ML & & & & & $\checkmark$ \\
\hline Head displacement, VT/ML & & & & & $\checkmark$ \\
\hline Pelvis displacement, VT/ML & & & & & $\checkmark$ \\
\hline Arm swing excursion & & & & & $\checkmark$ \\
\hline Joint kinematics (deg) & Nexus 2.6; Vicon, Oxford, UK & & & & \\
\hline Trunk flexion angle & & & & & $\checkmark$ \\
\hline Hip flexion/extension range & & & & & $\checkmark$ \\
\hline Knee flexion/extension range & & & & & $\checkmark$ \\
\hline Ankle dorsiflexion/plantar flexion range & & & & & $\checkmark$ \\
\hline $\begin{array}{l}\text { Joint angles (hip, knee, ankle) of stance } \\
\text { and swing leg at maximum toe clearance }\end{array}$ & & & & & $\checkmark$ \\
\hline Muscles activity (per muscle) & ZeroWire, Aurion Srl, Milan, Italy & & & & \\
\hline EMG onset and offset time & & & & & $\checkmark$ \\
\hline Duration of muscle activity & & & & & $\checkmark$ \\
\hline Total burst activation (integral of rectified EMG) & & & & & $\checkmark$ \\
\hline Time of peak muscle activation & & & & & $\checkmark$ \\
\hline \multicolumn{6}{|l|}{ Questionnaires } \\
\hline Balance confidence & Activities-specific Balance Confidence (ABC) Scale & $\checkmark$ & & & \\
\hline Freezing of gait (FOG) and falls & PD gait and falls questionnaire (FOG section) & $\checkmark$ & & & \\
\hline Physical activity & International Physical Activity Questionnaire (IPAQ) & $\checkmark$ & & & \\
\hline Quality of life & The 39-item Parkinson's Disease Questionnaire (PDQ-39) & $\checkmark$ & & & \\
\hline
\end{tabular}


Timed Up and Go (TUG) test Basic functional mobility will be assessed with the TUG test, providing the time required to rise from a chair, walk $3 \mathrm{~m}$ at a comfortable pace, turn, return to the chair and sit down [55]. In the present study, participants will complete a practice trial first. Two trials will then be completed and recorded; the second trial will include a dual task [26]. The participants will be asked to count backwards in threes from a randomly chosen number between 60 and 100 while performing the TUG test.

Electromyography Muscle activation will be recorded at $1 \mathrm{kHz}$ using the surface EMG bilateral ZeroWire system (ZeroWire, Aurion Srl, Milan, Italy). Recordings will be taken from the right and left lower limb muscles involved in gait: tibialis anterior, soleus, lateral and medial head of gastrocnemius, rectus femoris, biceps femoris, semitendinosus and vastus lateralis. Prior to applying the surface electrodes on the belly of the muscles of interest, participants with excessive hair over the muscles of interest will be shaved, using single-use disposal safety razors. Then, the skin will be cleaned thoroughly with a cotton ball and alcohol. This reduces impedance at the electrode-skin interface and improves the clarity of the myoelectric signal. After the skin is prepared, two Ambu surface electrodes (size $8 \times 22 / 30 \times 22$; electrode diameter $30 \times 20$; inter-electrode distance $25 \mathrm{~mm}$ ) will be placed on the muscles of interest according to the European recommendations for surface electromyography, Surface EMG for the Non-Invasive Assessment of Muscles (SENIAM) [56]. Muscle palpation will also be done to ensure validity of electrodes placement due to the anatomical variations between individuals.

\section{Intervention (a-tDCS and sham tDCS) during treadmill walking} The tDCS will be delivered using a portable battery-driven NeuroConn DC Plus stimulator. To ensure that the DC stimulator is not dropped, it is placed on the treadmill panel in front of the participant. Each participant will undergo two active a-tDCS sessions and one sham tDCS session with at least 1 week interval so that we avoid carry-over effects. The washout period in studies into PD and tDCS is varied between $48 \mathrm{~h}$ [57] to 1 week. The results of the statistical analysis for testing carry-over effects did not show any of these effects [57]. Therefore, a 1-week interval will be an appropriate washout period.

The anode will be placed centrally over the motor strip to cover a region $10-20 \%$ anterior to $\mathrm{Cz}$ during treadmill walking in all three sessions. To manage the risk of falls, participants will wear a harness if required. The position and size of the anode proposed in this study have been used by Kaski et al. [30, 33, 38] in a series of studies with positive effects on gait speed [30, 33, 38]. The cathode (either $10 \times 10$ or $4 \times 4 \mathrm{~cm}^{2}$ ) will be placed over the cerebellum, $2 \mathrm{~cm}$ below the inion on the median line, over the three sessions. The cathode placement in the two active tDCS sessions will be counterbalanced across the participants. Thus, one third of the participants will receive active tDCS with a large cathode first, and another one third of the participants will receive active tDCS with a small cathode electrode first; the final third of the participants will receive sham tDCS with either a small or large cathode electrode $(50 \%$ each). Electrodes will be inserted in saline-soaked sponge pockets. A cap and a strap will be used to attach the electrodes over the target locations. The current will be ramped up to $1 \mathrm{~mA}$ over $10 \mathrm{~s}$ in the active stimulation conditions and held constant for 20 min before ramping down over $10 \mathrm{~s}$ at the end of the stimulation [18]. In the sham condition, the current will be ramped up and down, at the beginning and at the end of the 20-min tDCS session (i.e. no active stimulation is administered in between). During both stimulation conditions (active and sham) all participants are asked to walk on a level Nautilus treadmill, and they will be allowed to use the hand rails. The principal researcher will apply the tDCS electrodes and oversee all aspects of the experiment. The tDCS will be terminated immediately if the participants become uncomfortable with it. The severity of adverse effects will be assessed using a scale suggested by Brunoni et al. [58] after each tDCS session.

\section{Blinding and randomization}

Blinding of the principal researcher will be achieved by using the "study mode" of the DC stimulator. The study mode activates active and sham stimulation with digital codes. To minimize biasing the outcomes, an independent researcher creates a list of participants using block randomization. A randomization sequence is produced through random permutations generated by a stochastic process program in $\mathrm{R}$ software version 3.3.4. The size of the blocks will be set to 3 with a ratio of $1: 1$ so that we need six blocks of the collection (1. ABC, 2. BAC, 3 . BCA, 4. CBA, 5. CAB, 6. ACB). We will draw six numbers randomly and sequentially from this collection.

In the randomization list the preprogrammed digital codes of tDCS and the size of the cathode electrode will be assigned to each participant for each session and submitted to the principal researcher via a sealed envelope. The independent researcher provides the digital code and cathode electrode size for the principal researcher prior to each tDCS session. The DC stimulator display continues to indicate the impedance and the time in the same fashion for both active and sham tDCS. The principal investigator will be blinded to the participant and experimental condition during the processing and analysing of the gait data. However, in the case of an adverse event, where it is necessary for the principal 
researcher to know which stimulation the participant is receiving, the principal researcher will be unblinded. Data from the identifiers will be removed and replaced by codes. However, the data will be re-identifiable by using the code or linking the participant's details to their allocation and to their different datasets. Reliability of blinding will also be assessed after each tDCS session by asking participants about the type of stimulation they believed they had received [58]. The principal investigator will also guess and record which stimulation type the participant had received.

\section{Modelling electric field intensity (EFI)}

We modelled the EFI using a computational model generated by a MATLAB toolbox, COMETS2, to simulate the two montages. Figure 3 compares the estimated EFI generated by the large anode and either a small or large cathode [59]. This illustrates that cerebellar activation is reduced using the $10 \mathrm{~cm} \times 10 \mathrm{~cm}$ cathode.

\section{Sample size}

In preparation for the development of the protocol, the required sample sizes were calculated using Power Analysis and $\mathrm{G}^{*}$ Power version 3.0. based on the primary outcome of gait velocity (measured by a 10 -m timed walk, Kaski et al. [30]) and considering previously published cut-points for clinically meaningful difference in gait speed in people with PD: 0.02, 0.06 and 0.1 for small, moderate and large changes respectively [60]. The effect size derived from their study reflects the difference between a-tDCS and sham tDCS combined with physical training on gait velocity. To detect the difference followed by Kaski and considering a significance level of $5 \%$, statistical power at $80 \%$, significance level of 0.05 and delta of 0.05 and allowing for $20 \%$ drop-out across the trial, the sample size was calculated to be 18 participants, which allows for a complete cross-over randomization. We will employ some strategies suggested in the study of Little et al. (2012) for limiting data missing during conducting the trial [61].

\section{Gait data processing}

The effects of the intervention on gait will be evaluated using a total of 21 gait parameters as listed in Table 1 [3]. The raw 3D position of each marker in GCS will be processed with the Vicon Nexus software to perform basic data processing (e.g. filtering at range of cut-off frequency of $5 \mathrm{~Hz}$, detection and selection of gait events) and extraction of gait characteristics (e.g. spatial and temporal) as well as linear and angular kinematic analyses (e.g. position and orientation of segments and the centre of mass). The first and last gait cycles will be discarded to avoid considering gait initiation and termination, leaving at least three complete gait cycles for each limb available for analysis.

The gait events, including heel-contact and toe-off, will be detected manually based on the vertical displacements of the calcaneus and second metatarsal head markers respectively. All spatiotemporal parameters will be calculated using conventional methods as described by Cole [3]. Maximum toe clearance will be defined as the highest vertical displacement of the toe relative to the ground during the swing phase [62].

Sagittal plane angular kinematics of the trunk, hip, knee and ankle joints will be assessed. Trunk angle is the angle between the vector joining the sacral and C7 markers and the vertical axis of the GCS. Hip angle is defined as the angle between the vector joining the hip and knee joints and the knee and ankle joints. Ankle angle is the angle between the vector joining the knee joint and the 2nd metatarsal joint, where zero degrees is the point at which the two vectors are in a vertical position [3]. To measure segmental control, the mediolateral and vertical displacement of the head and pelvis will be assessed. Arm swing excursion will be reported by

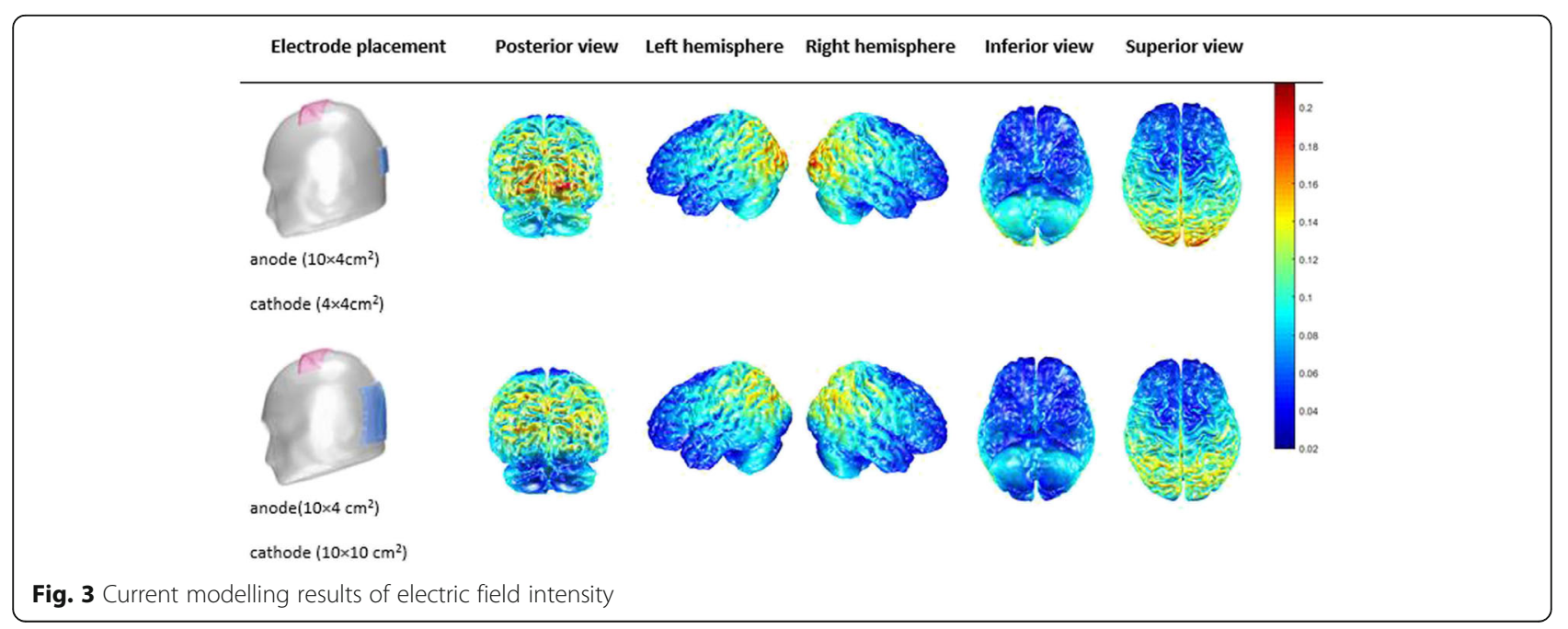


displacement of the wrists in the sagittal and frontal planes. All data in each gait cycle will be time-rescaled from 0 to 100 to facilitate averaging of all trials and reporting of events as a percentage of gait cycle. The gait data of each participant will be processed with the Vicon Nexus software, and all participants' gait data will be merged using a code written in MATLAB (R2017a; The Mathworks, Natick, MA, USA).

\section{EMG data processing}

All EMG data will be sampled at $1000 \mathrm{~Hz}$ and be filtered post-processing using a Butterworth fourth-order band pass filter $(10-500 \mathrm{~Hz})$. The EMG will be full wave rectified and normalized to the peak EMG recorded for each muscle. The EMG will then be aligned with the selected gait cycles and temporally normalized to $100 \%$ of the cycle. EMG will then be averaged across all gait cycles for the left and right sides. Such methods will reduce the subject-specific and situation-specific conditions that may result in signal variance. The activation profiles of each of the muscles will be assessed, including EMG onset and offset time; duration and timing of muscle activity; average time to peak muscle activation; and total burst activation (integral of rectified EMG).

The gait and EMG data processing will be conducted by the principal researcher, and all of the research team will have access to the final trial dataset.

\section{Statistical analysis}

Sample characteristics will be summarized into the number of non-missing data, mean, standard deviation, minimum, maximum and 95\% confidence interval, for quantitative variables. Number of non-missing data, frequency and proportion will be used for the description of categorical variables.

Gait speed will be the primary outcome in this study. The possible effects of participants' physical activity level at baseline on gait speed will be investigated by performing an analysis of the Pearson correlation between gait speed (estimated using the Vicon system before applying tDCS) and physical activity level.

We will examine the within-visit effects of each tDCS on gait speed as the primary outcome and the gait kinematics and TUG time as secondary outcomes using a paired $t$ test to compare pre- and post-tDSC measures.

The main effect of the three tDCS conditions (monopolar bilateral tDCS, bipolar bilateral tDCS and sham tDCS) on gait speed and gait kinematics will be examined through a linear mixed model (LMM) with random intercepts. The LMM model will contain participants nested in sequence as a random effect. In order to investigate the carry-over effect, we will compare three measures before the three tDCS procedures using one-way analysis of variance (ANOVA). When appropriate, post hoc comparisons will be carried out using a Tukey correction for multiple comparisons. The LMM will also be conducted to examine the potential interaction of time (levels: pre- and post-tDCS) and tDCS (levels: monopolar bilateral tDCS, bipolar bilateral tDCS and sham tDCS) in the two conditions of even and uneven surfaces. $P$ values less than 0.05 will be considered significant, and for valid interpretation of the results the confidence interval will be reported as an indication of effect size [63]. All analysis will be performed using SPSS software (version 23; IBM Corporation, Armonk, NY, USA). Missing data will be handled using a simple imputation method [58]. This Statistical Analysis Plan (SAP) will be provided before opening the database.

\section{Data management}

All data will be de-identified and coded. The electronic data will be stored on a secure university server which is regularly backed up and is password protected. The location on this server containing the computer files is password protected and is to be accessed only by research team members. All paper copies will be removed after a data collection session and stored in a locked cabinet in a secure, key-access laboratory at IHBI at QUT. The members of the research team, in particular those who are involved in data collection, will have the keys to access both the laboratory and the cabinets. According to the Australian Code for Responsible Conduct of Research (National Health and Medical Research Council (NHMRC), Australian Research Council (ARC), Universities Australia (UA), 2007), re-identifiable data will be stored for a minimum of 15 years after completion of the project. All digital research data will be held in a secure server at the IHBI with access only by investigators involved in the research based on QUT record management policy and the university's information privacy policy.

\section{Discussion}

During the last few years, the treatment of PD has shifted from a focus on pharmacological intervention towards investigation of different non-pharmacological approaches such as tDCS. Such approaches have been motivated due to the side effects of PD medications which have included dyskinesia $[28,64,65]$ and freezing of gait [2]. tDCS has been suggested as an alternative and promising adjunct treatment for PD. However, results of previous clinical trials in improving gait in $\mathrm{PD}$ have been equivocal, and an optimum tDCS montage has not been established. Therefore, we will systematically investigate the effects of two different montages administered during treadmill walking and their effects on gait and EMG parameters. To do so, we will use a modified montage used by Kaski [30, 33, 35, 38] to determine whether the synergetic effects of a-tDCS over both the 
premotor and primary motor cortices and c-tDCS over the cerebellum are more beneficial compared to the effects of a-tDCS over the premotor and primary motor cortices in PD. Furthermore, the comparison of the effects of the two montages on behavioural changes will provide information for further establishing the exact role of a-tDCS with a large electrode (monopolar set up) and a-tDCS with small cathode electrode (bipolar set-up) on gait in PD. Indeed, our initial evaluation of EFI modelling (Fig. 3) shows that the small cathode electrode over the cerebellum results in more pronounced current flow to the cerebellum, affecting its posterior, inferior and lateral aspects and the vermis of the cerebellum, while the EFI over the cerebellum with the large cathode electrode is on the lateral edges of the cerebellum.

One of the possible methodological limitations of this study will be the potential of carry-over effects between testing sessions. Consequently, we will compare the pre-test results of all conditions to ensure they are not significantly different due to possible carry-over effects. Also, we will use a self-selected most comfortable walking speed on the treadmill, which varies in each participant and might result in different activation of M1. Furthermore, we will only assess the acute effects of tDCS on the outcome measures, as long-term effects of a-tDCS on gait abilities were deemed beyond the scope of this study. Nonetheless, this study will inform future studies on the effects of optimal montage of tDCS combined with treadmill walking. If our proposed montage is effective in improving gait in $\mathrm{PD}$, further research needs to be conducted to identify the long-term effects of multiple sessions of tDCS with the same parameters and concurrent accumulation effects of tDCS with other physical tasks. This may offer a significant option for the treatment of PD.

\section{Trial status}

This trial is an ongoing project started in 2017 and is expected to be completed in November 2018.

\section{Additional file}

Additional file 1: SPIRIT checklist. (DOCX $56 \mathrm{~kb}$ )

\footnotetext{
Abbreviations

ABC: Activities-specific Balance Confidence (Scale); ACE: Addenbrooke's Cognitive Examination; a-tDCS: Anodal tDCS; EMG: Electromyography; GC: Gait cycle; GCS: Global Coordinate System; IPAQ: International Physical Activity Questionnaire; LMM: Linear mixed model; M1: Primary motor cortex; PD: Parkinson's disease; PDQ-39: Parkinson's Disease Questionnaire; PQI: Parkinson's Queensland Incorporated; QUT: Queensland University of Technology; tDCS: Transcranial direct current stimulation; TMS: Transcranial magnetic stimulation; TUG: Timed Up and Go (test); UPDRS: Unified Parkinson's Disease Rating Scale
}

\section{Acknowledgements}

The authors of this paper acknowledge their gratitude to the participants in this research project

\section{Funding}

This work was supported by the Queensland University of Technology (QUT). VA is the recipient of a QUT Higher Degree Research (HDR) Tuition Fee Sponsorship and Faculty/School Top Up Scholarship and a salary from the University of Social Welfare and Rehabilitation Sciences. This study will be designed and undertaken independently from the funders of the project.

\section{Availability of data and materials}

Access to the full protocol and participant-level dataset may be provided upon request to the authors.

\section{Authors' contributions}

VA wrote the manuscript. VA, MM and GK contributed to the design of the randomized controlled trial (RCT). GK and LF contributed to the design of acquisition, analysis and reporting of gait data. VA, GK and RP are responsible for reasonable statistical analysis. SS and all other authors critically read, improved and approved the final manuscript.

\section{Ethics approval and consent to participate}

The QUT Human Research Ethics Committee has approved this study (1500001094). All participants must provide a written informed consent form to participate in the study.

Consent for publication

Not applicable.

\section{Competing interests}

The authors declare that they have no competing interests.

\section{Publisher's Note}

Springer Nature remains neutral with regard to jurisdictional claims in published maps and institutional affiliations.

\section{Author details}

'Movement Neuroscience Program, Institute of Health and Biomedical Innovation, Queensland University of Technology, Brisbane, QLD, Australia. ${ }^{2}$ Iranian Research Centre on Ageing, The University of Social Welfare and rehabilitation Sciences, Tehran, Iran. ${ }^{3}$ Centre for Clinical Research, The University of Queensland, Brisbane, QLD, Australia. ${ }^{4}$ Institute of Health and Biomedical Innovation Queensland University of Technology, Brisbane, QLD, Australia. ${ }^{5}$ School of Exercise and Nutrition Sciences, Queensland University of Technology, Brisbane, QLD, Australia. ${ }^{6}$ Institute for Social Science Research (ISSR), The University of Queensland, Brisbane, QLD, Australia.

Received: 27 March 2018 Accepted: 13 October 2018

Published online: 29 November 2018

References

1. Mirelman A, Bernad-Elazari H, Thaler A, Giladi-Yacobi E, Gurevich T, GanaWeisz $M$, et al. Arm swing as a potential new prodromal marker of Parkinson's disease. Movement Disorders. 2016;31(10):1527-34.

2. Nutt JG, Wooten GF. Diagnosis and initial management of Parkinson's disease. N Engl J Med. 2005;353(10):1021-7.

3. Cole MH, Silburn PA, Wood JM, Worringham CJ, Kerr GK. Falls in Parkinson's disease: kinematic evidence for impaired head and trunk control. Movement Disorders. 2010;25(14):2369-78.

4. Mitoma H, Hayashi R, Yanagisawa N, Tsukagoshi H. Characteristics of parkinsonian and ataxic gaits: a study using surface electromyograms, angular displacements and floor reaction forces. J Neurol Sci. 2000;174(1):22-39.

5. Hiraoka K, Matsuo Y, Abe K. Soleus H-reflex inhibition during gait initiation in Parkinson's disease. Movement Disorders. 2005;20(7):858-64.

6. Hong M, Perlmutter JS, Earhart GM. A kinematic and electromyographic analysis of turning in people with Parkinson disease. Neurorehabil Neural Repair. 2009;23(2):166-76.

7. Rietdijk CD, Perez-Pardo P, Garssen J, van Wezel RJ, Kraneveld AD. Exploring Braak's hypothesis of Parkinson's disease. Front Neurol. 2017;8:37.

8. Buhmann C, Glauche V, Sturenburg HJ, Oechsner M, Weiller C, Buchel C. Pharmacologically modulated $\mathrm{fMRI}$ — cortical responsiveness to levodopa in drug-naive hemiparkinsonian patients. Brain. 2003;126(Pt 2):451-61.

9. Di Stasio F, Suppa A, Fabbrini A, Marsili L, Asci F, Conte A, et al. Parkinsonism is associated with altered primary motor cortex plasticity in 
frontotemporal dementia—primary progressive aphasia variant. Neurobiol Aging. 2018;69:230-8.

10. Martinu K, Monchi O. Cortico-basal ganglia and cortico-cerebellar circuits in Parkinson's disease: pathophysiology or compensation? Behav Neurosci. 2013;127(2):222-36.

11. Wu T, Hallett M. The cerebellum in Parkinson's disease. Brain. 2013;136(3):696-709.

12. Nitsche MA, Paulus W. Excitability changes induced in the human motor cortex by weak transcranial direct current stimulation. J Physiol. 2000;527(Pt 3):633-9.

13. Nitsche MA, Paulus W. Sustained excitability elevations induced by transcranial DC motor cortex stimulation in humans. Neurology. 2001;57(10):1899-901.

14. Daskalakis ZJ, Paradiso GO, Christensen BK, Fitzgerald PB, Gunraj C, Chen R. Exploring the connectivity between the cerebellum and motor cortex in humans. J Physiol. 2004;557(Pt 2):689-700.

15. Miniussi C, Harris JA, Ruzzoli M. Modelling non-invasive brain stimulation in cognitive neuroscience. Neurosci Biobehav Rev. 2013;37(8):1702-12.

16. Perceval G, Floel A, Meinzer M. Can transcranial direct current stimulation counteract age-associated functional impairment? Neurosci Biobehav Rev. 2016;65:157-72.

17. Bikson M, Grossman P, Thomas C, Zannou AL, Jiang J, Adnan T, et al. Safety of transcranial direct current stimulation: evidence based update 2016. Brain Stimulation. 2016;9(5):641-61.

18. Fregni F, Nitsche MA, Loo CK, Brunoni AR, Marangolo P, Leite J, et al. Regulatory considerations for the clinical and research use of transcranial direct current stimulation (tDCS): review and recommendations from an expert panel. Clin Res Regul Aff. 2015;32(1):22-35.

19. Stagg CJ, Nitsche MA. Physiological basis of transcranial direct current stimulation. Neuroscientist. 2011;17(1):37-53.

20. Fertonani A, Miniussi C. Transcranial electrical stimulation: what we know and do not know about mechanisms. Neuroscientist. 2016;23(2):109-23.

21. Nitsche MA, Cohen LG, Wassermann EM, Priori A, Lang N, Antal A, et al. Transcranial direct current stimulation: state of the art 2008. Brain Stimulation. 2008;1 (3):206-23.

22. Schulz R, Gerloff C, Hummel FC. Non-invasive brain stimulation in neurological diseases. Neuropharmacology. 2013;64:579-87.

23. Ferrucci R, Mameli F, Ruggiero F, Priori A. Transcranial direct current stimulation as treatment for Parkinson's disease and other movement disorders. Basal Ganglia. 2016;6(1):53-61.

24. Costa-Ribeiro A, Maux A, Bosford T, Aoki Y, Castro R, Baltar A, et al. Transcranial direct current stimulation associated with gait training in Parkinson's disease: a pilot randomized clinical trial. Developmental Neurorehabil. 2017;20(3):121-8.

25. Costa-Ribeiro A, Maux A, Bosford T, Tenorio Y, Marques D, Carneiro M, et al. Dopamine-independent effects of combining transcranial direct current stimulation with cued gait training on cortical excitability and functional mobility in Parkinson's disease. J Rehabil Med. 2016:48(9):819-23.

26. Schabrun SM, Lamont RM, Brauer SG. Transcranial direct current stimulation to enhance dual-task gait training in Parkinson's disease: a pilot RCT. PLOS One. 2016;11(6):e0158497.

27. von Papen M, Fisse M, Sarfeld AS, Fink GR, Nowak DA. The effects of $1 \mathrm{~Hz}$ rTMS preconditioned by tDCS on gait kinematics in Parkinson's disease. J Neural Transm. 2014;121(7):743-54.

28. Benninger DH, Lomarev M, Lopez G, Wassermann EM, Li X, Considine E, et al. Transcranial direct current stimulation for the treatment of Parkinson's disease. J Neurol Neurosurg Psychiatry. 2010;81(10):1105-11.

29. Valentino F, Cosentino G, Brighina F, Pozzi NG, Sandrini G, Fierro B, et al. Transcranial direct current stimulation for treatment of freezing of gait: a cross-over study. Movement Disorders. 2014;29(8):1064-9.

30. Kaski D, Dominguez RO, Allum JH, Islam AF, Bronstein AM. Combining physical training with transcranial direct current stimulation to improve gait in Parkinson's disease: a pilot randomized controlled study. Clin Rehabil. 2014:28(11):1115-24.

31. Verheyden G, Purdey J, Burnett M, Cole J, Ashburn A. Immediate effect of transcranial direct current stimulation on postural stability and functional mobility in Parkinson's disease. Movement Disorders. 2013;28(14):2040-1.

32. Fernandez-Lago H, Bello O, Mora-Cerda F, Montero-Camara J, Fernandez-DelOlmo MA. Treadmill walking combined with anodal transcranial direct current stimulation in Parkinson disease: a pilot study of kinematic and neurophysiological effects. Am J Physical Medicine Rehabil. 2017;96(11):801-8.

33. Kaski D, Allum JH, Bronstein AM, Dominguez RO. Applying anodal tDCS during tango dancing in a patient with Parkinson's disease. Neurosci Lett. 2014;568:39-43.
34. Yotnuengnit $P$, Bhidayasiri R, Donkhan R, Chaluaysrimuang J, Piravej K. Effects of transcranial direct current stimulation plus physical therapy on gait in patients with Parkinson disease: a randomized controlled trial. Am J Physical Medicine Rehabil. 2018:97(1):7-15.

35. Kaski D, Bronstein AM. Treatments for neurological gait and balance disturbance: the use of noninvasive electrical brain stimulation. Advances Neurosci. 2014;2014:1-13.

36. Chen R. Transcranial direct current stimulation as a treatment for Parkinson's disease-interesting, but not ready for prime time. J Neurol Neurosurg Psychiatry. 2010;81(10):1061.

37. McNeely ME, Duncan RP, Earhart GM. Differential effects of tango, treadmill, and stretching interventions on gait in people with Parkinson's disease, 20th International Congress of Parkinson's Disease and Movement Disorders. Berlin: International Parkinson and Movement Disorder Society; 2016.

38. Kaski D, Quadir S, Patel M, Yousif N, Bronstein AM. Enhanced locomotor adaptation aftereffect in the "broken escalator" phenomenon using anodal tDCS. J Neurophysiol. 2012;107(9):2493-505.

39. Poortvliet P, Hsieh B, Cresswell A, Au J, Meinzer M. Cerebellar transcranial direct current stimulation improves adaptive postural control. Clin Neurophysiol. 2018;129(1):33-41.

40. Mioshi E, Dawson K, Mitchell J, Arnold R, Hodges JR. The Addenbrooke's Cognitive Examination Revised (ACE-R): a brief cognitive test battery for dementia screening. Int J Geriatr Psychiatry. 2006;21(11):1078-85.

41. Goetz CG, Tilley BC, Shaftman SR, Stebbins GT, Fahn S, Martinez-Martin P, et al. Movement Disorder Society-sponsored revision of the Unified Parkinson's Disease Rating Scale (MDS-UPDRS): scale presentation and clinimetric testing results. Mov Disord. 2008;23(15):2129-70.

42. Mantyjarvi M, Laitinen T. Normal values for the Pelli-Robson contrast sensitivity test. J Cataract Refract Surg. 2001;27(2):261-6.

43. Haymes SA, Chen J. Reliability and validity of the Melbourne Edge Test and High/Low Contrast Visual Acuity chart. Optom Vis Sci. 2004;81(5):308-16.

44. Bailey IL, Lovie JE. New design principles for visual acuity letter charts. Am J Optom Physiol Optic. 1976;53(11):740-5.

45. Bailey IL, Lovie-Kitchin JE. Visual acuity testing. From the laboratory to the clinic. Vis Res. 2013;90:2-9.

46. Nolano M, Provitera V, Estraneo A, Selim MM, Caporaso G, Stancanelli A, et al. Sensory deficit in Parkinson's disease: evidence of a cutaneous denervation. Brain. 2008;131(Pt 7):1903-11.

47. Kegelmeyer DA, Kloos AD, Thomas KM, Kostyk SK. Reliability and validity of the Tinetti Mobility Test for individuals with Parkinson disease. Phys Ther. 2007:87(10):1369-78.

48. Wang C, Chen P, Zhuang J. Validity and reliability of International Physical Activity Questionnaire-Short Form in Chinese youth. Res Q Exerc Sport. 2013;84(Suppl 2):S80-6.

49. Franchignoni F, Giordano A, Ronconi G, Rabini A, Ferriero G. Rasch validation of the Activities-specific Balance Confidence Scale and its short versions in patients with Parkinson's disease. J Rehabil Med. 2014;46(6):532-9.

50. Jenkinson C, Fitzpatrick R, Peto V, Greenhall R, Hyman N. The Parkinson's Disease Questionnaire (PDQ-39): development and validation of a Parkinson's disease summary index score. Age Ageing. 1997;26(5):353-7.

51. Giladi N, Shabtai H, Simon ES, Biran S, Tal J, Korczyn AD. Construction of freezing of gait questionnaire for patients with Parkinsonism. Parkinsonism Relat Disord. 2000:6(3):165-70.

52. Goetz CG, Poewe W, Rascol O, Sampaio C, Stebbins GT, Counsell C, et al. Movement Disorder Society Task Force report on the Hoehn and Yahr staging scale: status and recommendations. Mov Disord. 2004;19(9):1020-8.

53. Levine D, Richards J, Whittle MW. Whittle's gait analysis. 5th ed. London, Edinburgh: Churchill Livingstone/Elsevier; 2012.

54. Perry JB, Judith M. Gait analysis: normal and pathological function. 2nd ed. Thorofare: SLACK; 2010.

55. da Silva BA, Faria C, Santos MP, Swarowsky A. Assessing Timed Up and Go in Parkinson's disease: reliability and validity of Timed Up and Go Assessment of biomechanical strategies. J Rehabil Med. 2017;49(9):723-31.

56. Hermens HJ, Freriks B, Disselhorst-Klug C, Rau G. Development of recommendations for SEMG sensors and sensor placement procedures. J Electromyogr Kinesiol. 2000;10(5):361-74.

57. Fregni F, Boggio PS, Santos MC, Lima M, Vieira AL, Rigonatti SP, et al. Noninvasive cortical stimulation with transcranial direct current stimulation in Parkinson's disease. Mov Disord. 2006;21(10):1693-702.

58. Brunoni AR, Amadera J, Berbel B, Volz MS, Rizzerio BG, Fregni F. A systematic review on reporting and assessment of adverse effects 
associated with transcranial direct current stimulation. Int J Neuropsychopharmacol. 2011;14(8):1133-45.

59. Lee C, Jung YJ, Lee SJ, Im CH. COMETS2: An advanced MATLAB toolbox for the numerical analysis of electric fields generated by transcranial direct current stimulation. J Neurosci Methods. 2017;277:56-62.

60. Hass CJ, Bishop M, Moscovich M, Stegemoller EL, Skinner J, Malaty IA, et al. Defining the clinically meaningful difference in gait speed in persons with Parkinson disease. J Neurol Phys Ther. 2014;38(4):233-8.

61. Little RJ, D'Agostino R, Cohen ML, Dickersin K, Emerson SS, Farrar JT, et al. The prevention and treatment of missing data in clinical trials. N Engl J Med. 2012;367(14):1355-60.

62. Winter DA. The biomechanics and motor control of human gait: normal, elderly and pathological. 2nd ed. Waterloo: University of Waterloo Press; 1991.

63. Wasserstein RL, Lazar NA. The ASA's statement on p-values: context, process, and purpose. Am Stat. 2016;70(2):129-33.

64. Olanow CW, Watts RL, Koller WC. An algorithm (decision tree) for the management of Parkinson's disease (2001): treatment guidelines. Neurology. 2001;56(11 Suppl 5):S1-S88.

65. Jankovic J. Complications and limitations of drug therapy for Parkinson's disease. Neurology. 2000;55(12 Suppl 6):S2-6.

Ready to submit your research? Choose BMC and benefit from:

- fast, convenient online submission

- thorough peer review by experienced researchers in your field

- rapid publication on acceptance

- support for research data, including large and complex data types

- gold Open Access which fosters wider collaboration and increased citations

- maximum visibility for your research: over $100 \mathrm{M}$ website views per year

At $\mathrm{BMC}$, research is always in progress.

Learn more biomedcentral.com/submissions 